\title{
THREE-DIMENSIONAL PHOTONIC CRYSTALS
}

\section{Microscale holography}

Three-dimensional (3D) photonic crystals created by a simple form of holography are now available at telecommunications wavelengths, thanks to researchers at the University of Texas at Austin (Appl. Phys. Lett. 90, 093102; 2007).

Multiple reflections of waves in a periodic structure can create so-called standing waves, which do not propagate through the medium. The structural periodicity must be on a similar length scale to the wavelength, a condition met for a range of wave frequencies referred to as a bandgap. The concept will be familiar to anyone who has studied semiconductors - atomic spacing on the order of the de Broglie wavelength of the 'free electrons' opens up an electronic bandgap, giving silicon many important properties. Optical or photonic bandgap materials offer unprecedented control of light: examples include waveguides with almost no loss - even at sharp bends - as well as a whole host of miniature optical components, presenting the possibility of the optical equivalent of integrated (electronic) circuits.

But how best to fabricate these intricate $3 \mathrm{D}$ crystals? For visible light, the periodicity must be on the submicrometre scale, and any imperfections will probably degrade the desired material properties. Holography offers a solution. Multiple laser beams can generate a complex interference pattern. Create this pattern in a

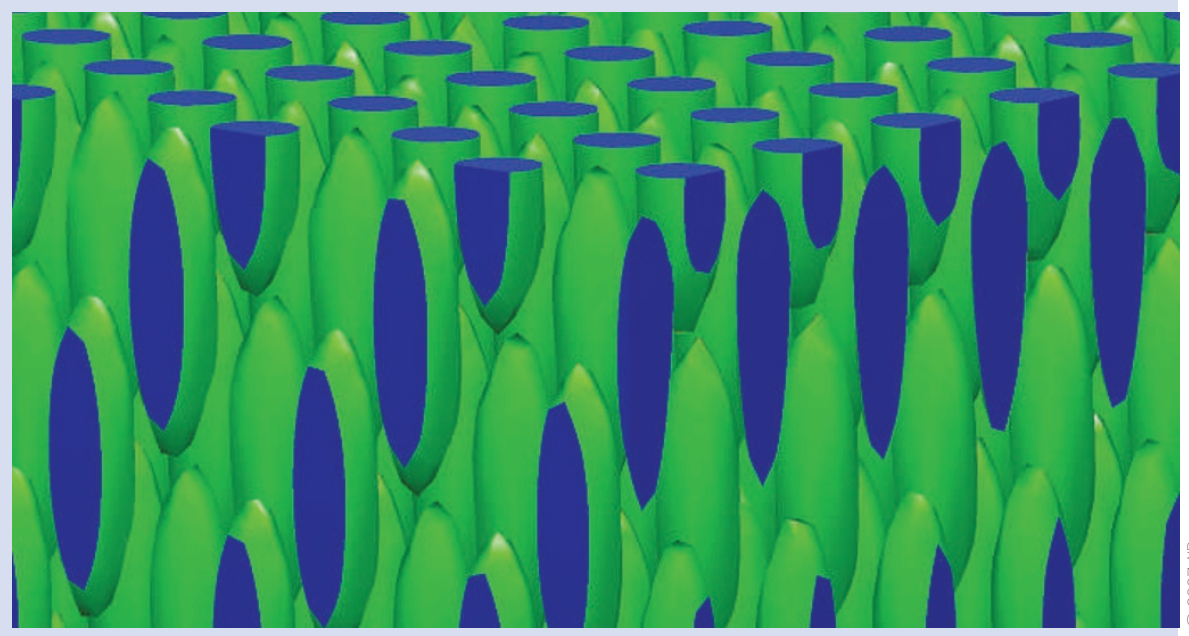

photosensitive polymer and, just like a $3 \mathrm{D}$ version of standard photography, some parts of the polymer become 'soluble' and some don't. The holographic interference pattern is transferred to the polymer by placing it in a suitable developer. The approach taken by Jiaqi Chen and colleagues uses a prism to create four beams from a single laser source - less complicated and requiring less stabilization than using independent components for each beam. Using ultraviolet laser light, the team create a material with a bandgap centred on a wavelength of $1.5 \mu \mathrm{m}$. The technique has been demonstrated before, but to make materials with a bandgap at longer wavelengths. A shorter wavelength requires a smaller periodicity, complicating the fabrication.

The drawback is that the light-sensitive materials required for this holography may not always be the best in terms of optical or mechanical properties. An approach investigated over the past few years, although not yet implemented in this case, is to use the holographically defined structure as a template - filling the voids with another material with more ideal characteristics, such as a high refractive index or strong electro-optic properties. A combination of these two techniques could enable construction of materials with predetermined optical properties, allowing intricate control over the flow of light.

David Gevaux 